\title{
A experiência de adaptação de mães de crianças e adolescentes com paralisia cerebral: Um estudo qualitativo
}

\author{
Inês Mimoso', Luciana Sotero², Ana Isabel Cunha ${ }^{3}$ e Juliana Queiroz ${ }^{4}$ \\ ${ }^{1}$ Faculdade de Psicologia e de Ciências da Educação, Universidade de Coimbra, Portugal | \\ inesmimoso400@gmail.com | http://orcid.org/0000-0002-6066-1375 \\ ${ }^{2}$ Centro de Estudos Sociais, Faculdade de Psicologia e de Ciências da Educação da \\ Universidade de Coimbra, Portugal | lucianasotero@fpce.uc.pt | https://orcid.org/0000-0001- \\ 8393-2775 \\ ${ }^{3}$ Departamento de Psicologia e Educação, Universidade da Beira Interior, Portugal | \\ acunha@ubi.pt | http://orcid.org/0000-0002-0515-707X \\ ${ }^{4}$ Centro de Ciências Biológicas e da Saúde, Universidade do Estado do Pará, Brasil \\ comportamentomotor@hotmail.com | https://orcid.org/0000-0003-1931-933X
}

Resumo: Introdução: A paralisia cerebral (PC) é uma das condições crónicas pediátricas mais comuns, impondo múltiplos desafios à vida das famílias, em particular, às mães, que assumem a maior parte dos cuidados da criança. No entanto, apesar das exigências associadas ao cuidado de uma criança com PC, a literatura tem demonstrado que os pais também possuem capacidades para enfrentar a adversidade. Objetivos: Compreender a experiência destas mães, explorando os fatores que, na sua perceção, contribuem para uma adaptação positiva. Métodos: Foi realizado um estudo qualitativo, com recurso a entrevistas semiestruturadas a 6 mães de crianças/adolescentes com PC. Os dados foram analisados recorrendo a uma análise temática. Resultados: Identificaram-se cinco grandes temas que evidenciam fatores importantes para a adaptação: (1) cooperação intrafamiliar; (2) rede de apoio; (3) perceção de competência; (4) pôr a PC "no seu lugar"; e (5) manutenção de uma visão positiva. Conclusões: A identificação destes temas permitiu evidenciar as forças e os recursos associados a uma trajetória adaptativa de mães de crianças/adolescentes com PC traduzindo-se em pistas para a intervenção clínica e um ponto de partida para futuras investigações.

Palavras-chave: Paralisia Cerebral; Mães; Adaptação; Análise Temática.

The Adaptation Experience of Mothers of Children and Adolescents with Cerebral Palsy: A Qualitative Study

\begin{abstract}
Introduction: Cerebral palsy (CP) is one of the most common chronic pediatric conditions worldwide, raising multiple challenges on the family, particularly, on mothers, who assume most of the care that the child needs. Nevertheless, despite the demands associated with the care of the child, the literature has been showing that parents also have the competence to face adversity. Goals: Understand the experience of these mothers, exploring the factors that, in their perception, contribute to a positive adaptation. Methods: A qualitative study was conducted, using semi-structured interviews, with six mothers of children/adolescents with CP. The present data were analysed using a thematic analysis. Results: Five major themes were identified that highlight important factors for the adaptation: (1) intrafamily cooperation; (2) support network; (3) perception of competence; (4) put the PC "in its place"; e (5) maintenance of a positive outlook. Conclusions: The identification of these themes allows us to highlight the strengths and resources associated with an adaptive trajectory of mothers of children/adolescents with $\mathrm{CP}$, translating into clues for clinical intervention and a starting point for future investigations.
\end{abstract}

Keywords: Cerebral Palsy; Mothers; Adaptation; Thematic Analysis.

\section{Introdução}

A paralisia cerebral (PC) é uma condição neurodesenvolvimental, com início na primeira infância e que persiste ao longo da vida (Pousada et al., 2013), sendo considerada uma das condições crónicas pediátricas mais comuns (Eunson, 2016). Por ser uma condição complexa e multifatorial, as crianças/adolescentes com PC requerem cuidados acrescidos, para além dos normativos típicos do seu desenvolvimento (Raina et al., 2005). 
Surge, assim, um novo papel no seio familiar - o de cuidador principal - ou seja, aquele que assegura a maior parte dos cuidados que a criança necessita (Santos et al., 2010), assistindo-se, muitas vezes, a uma sobreposição entre o papel parental e o de cuidador (Dixon-Wood et al., 2002). Segundo a literatura, as mães são, maioritariamente, quem assume o papel de cuidador principal (Macedo et al., 2015).

A PC constitui um stressor significativo para os pais (Cunha et al., 2017; Pousada et al., 2013), pelo que se torna fundamental compreender e identificar os fatores que contribuem para a sua adaptação. O modelo de resiliência familiar (Walsh, 2016) representa um importante contributo para compreender a adaptação face a acontecimentos persistentes de stress, reconhecendo que processos familiares, ao nível do sistema de crenças, dos padrões organizacionais e dos processos de comunicação, são promotores de uma adaptação positiva. Vários estudos reportam diversos fatores que contribuem para o processo de adaptação dos pais a uma condição crónica num(a) filho(a), nomeadamente, as redes de apoio do sistema (Gomes et al., 2019; Santos et al., 2019), a perceção de competência e autoestima dos pais (Guillamón et al., 2013), a importância de manter uma atitude positiva face à adversidade e o apoio espiritual (Santos et al., 2019).

No contexto português, a revisão da literatura permitiu verificar a escassez de estudos, (sobretudo qualitativos), sobre a experiência de adaptação parental à PC. Assim, este estudo emerge do interesse em compreender a experiência de mães de crianças/adolescentes com PC, explorando os fatores que, na sua perceção, contribuem para uma adaptação positiva. Assim, foi formulada a seguinte questão de investigação: Como é que as mães descrevem o que foi e tem sido importante para lidar positivamente com a PC do(as) filho(as)?

\section{Metodologia}

\subsection{Procedimentos}

Este estudo insere-se num projeto de investigação transcultural sobre pais-cuidadores portugueses e brasileiros de crianças/adolescentes com PC. Os critérios de inclusão para a subamostra portuguesa foram: ser mãe/pai de uma criança/adolescente com PC (máximo 18 anos) e frequentar uma Associação de Paralisia Cerebral (APC). Integraram a investigação, numa fase quantitativa, 186 pais. Destes, seis foram selecionados aleatoriamente e convidados a participar neste estudo qualitativo.

Foram realizadas entrevistas individuais semiestruturadas, a partir de um guião elaborado pelas autoras, baseado num trabalho de investigação sobre adaptação familiar à doença crónica pediátrica (Cunha, 2011). Foi sugerido às participantes que imaginassem estar a contar uma história, num livro intitulado "A minha história como cuidadora de um(a) filho(a) com PC", dividido em três capítulos: 1) o primeiro centrado no momento em que descobriu a PC do(a) filho(a); 2) o segundo baseado no que fez e tem feito para lidar com a PC, e 3) o terceiro focado em como se sente no momento atual. No âmbito do presente trabalho serão apresentados os dados relativos à perceção de adaptação das mães (capítulos $2 \mathrm{e}$ 3). As entrevistas foram realizadas pela investigadora principal, entre fevereiro e março de 2020, gravadas em formato áudio e transcritas de forma integral, tendo, em média, a duração de 28 minutos. A investigadora tinha ao seu dispor uma sala reservada, cedida pela APC onde foi realizado o estudo.

O estudo foi aprovado pela Comissão de Ética e Deontologia da Investigação da Faculdade de Psicologia e de Ciências da Educação da Universidade de Coimbra (parecer no 1.811.549). Os participantes foram informados acerca dos objetivos do estudo e das questões éticas, tendo sido solicitado o consentimento informado por escrito. 


\subsection{Participantes}

A amostra é constituída por seis mães. A informação sobre as participantes e filhos(as) encontra-se descrita na Tabela 1.

Tabela 1. Informação sobre as participantes.

\begin{tabular}{|c|c|c|c|c|c|c|c|}
\hline Id. ${ }^{1}$ & Idade & $\begin{array}{l}\text { Situação } \\
\text { Relacional }\end{array}$ & Escolaridade & $\begin{array}{l}\text { Situação } \\
\text { Profissional }\end{array}$ & $\begin{array}{c}\text { Sexo } \\
\text { do(a) } \\
\text { filho(a) }\end{array}$ & $\begin{array}{l}\text { Idade } \\
\text { do(a) } \\
\text { filho(a) }\end{array}$ & $\begin{array}{c}\text { Severidade } \\
\text { da } \mathrm{PC}^{2}\end{array}$ \\
\hline I. & 50 & Casada & $12^{\circ}$ ano & Doméstica & M & 17 & V \\
\hline M. & 30 & Casada & Licenciatura & Empregada & $M$ & 4 & II \\
\hline $\mathrm{H}$. & 43 & Divorciada & Mestrado & Empregada & M & 8 & I \\
\hline P. & 49 & Casada & $9^{\circ}$ ano & Empregada & M & 17 & I \\
\hline C. & 37 & Casada & $9^{\circ}$ ano & Doméstica & $\begin{array}{c}\mathrm{M} / \mathrm{M} \\
\text { (gémeos) }\end{array}$ & 6 & I \\
\hline $\mathrm{R}$. & 42 & Recasada & $6^{\circ}$ ano & Doméstica & $\mathrm{F}$ & 15 & 1 \\
\hline
\end{tabular}

${ }^{1}$ As iniciais são fictícias, de forma a salvaguardar o anonimato das participantes.

${ }^{2} \mathrm{~A}$ severidade da PC foi avaliada segundo o Gross Motor Function Classification System (GMFCS), que caracteriza as incapacidades motoras. Divide-se em 5 níveis, variando desde a independência nos movimentos (nível I) à completa dependência do cuidador (nível V) (GMFCS; Palisano et al., 2008).

\subsection{Análise dos Dados}

Os dados foram analisados recorrendo a uma análise temática. Este método permite identificar, analisar e interpretar padrões repetidos de significado, a partir dos dados qualitativos recolhidos (Braun \& Clarke, 2006). Seguindo os passos propostos pelos autores, a primeira etapa consistiu na familiarização com os dados, facilitada pelo processo de transcrição e leitura aprofundada das entrevistas. De seguida, procedeu-se à criação de códigos iniciais, produzindo-se um conjunto alargado de códigos, que resultou na necessidade de fusão de algumas categorias que, apesar de terem uma designação diferente, refletiam a mesma ideia (e.g., apoio dos técnicos e apoio dos profissionais). A criação dos códigos iniciais foi feita de forma independente por dois elementos da equipa de investigação. Procedeu-se, de seguida, à reorganização dos códigos, agrupando-os em categorias mais abrangentes (temas e subtemas provisórios). Nesta etapa, as categorias criadas dependeram da sua pertinência para o estudo, e não da sua frequência ao longo das entrevistas. Neste processo de reorganização, participaram todos os elementos da equipa de investigação, o que promoveu a discussão e o refinamento dos temas propostos. No final, procedeu-se, novamente, à revisão dos temas e leitura das entrevistas, de forma a verificar a coerência e pertinência quer dos temas, quer das transcrições que os ilustram. As designações provisórias de cada tema e subtema foram sendo apuradas, através de sucessivas reuniões entre os elementos da equipa. 
Vol. 8 | Investigação Qualitativa em Saúde: Avanços e Desafios

\section{Resultados}

Da análise efetuada emergiram cinco temas e onze subtemas que procuram refletir a experiência de adaptação das mães (Figura 1).

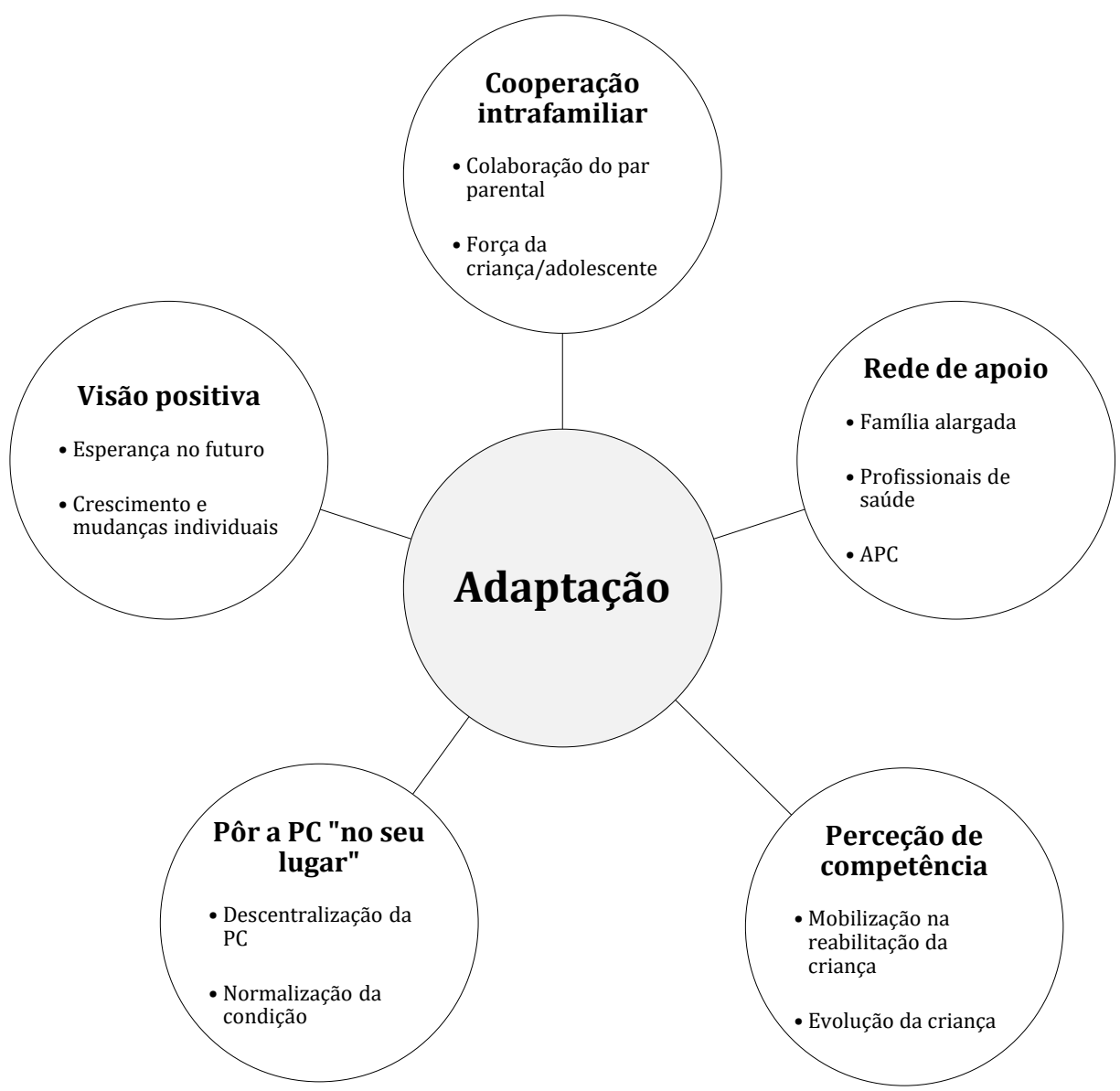

Fig. 1. Mapa temático relativo à adaptação das mães.

O tema da "Colaboração intrafamiliar" foi recorrente ao longo das entrevistas, refletindo a forma como as participantes percecionam a importância do suporte mútuo na família nuclear, nomeadamente no sistema parental, e enfatizam a ajuda da própria criança no processo de adaptação. De facto, apesar das dificuldades, a forte ligação entre os membros do casal em torno de um "projeto comum" emerge como uma força para lidar com a PC:

"Enquanto casal tivemos um período difícil, nunca esteve em causa não nos entendermos e não seguirmos isto como um projeto comum, sempre foi um projeto comum dos dois" (M.|filho|4A).

Outra mãe referiu que, apesar de estar divorciada do pai do filho, a prioridade sempre foi o bem-estar e as necessidades da criança, evidenciando o esforço parental nesse sentido:

"Eu e o pai, neste momento estamos separados, na altura estávamos juntos, mas não interessa, é a mesma coisa. Eu e o pai sempre fomos pessoas preocupadas e pronto, interventivas" ( $\mathrm{H}$.|filho|8A). 
Foram também mencionados aspetos relacionados com a criança que facilitaram o processo de adaptação, nomeadamente, a força que os(as) filhos(as) transmitem:

"Mas também já dei com ele a treinar em frente ao espelho, a correr igual aos jogadores de futebol, (...) acho que eles às vezes também são uma grande força da natureza e de facto o $B$. ensinou-me isso (...) a condição dele é um handicap, mas ele não desiste e, portanto, se ele não desiste nós também não vamos desistir." (M.|filho|4A).

O tema "Rede de apoio" demonstra como o apoio da família alargada, dos profissionais de saúde e da instituição de apoio constitui um recurso importante para as mães. Quanto ao apoio da família, os avós e/ou os padrinhos foram referidos como "pilares" importantes para lidar com as exigências da condição, nomeadamente, em termos materiais e emocionais:

"Nós temos a sorte, o B. tem a sorte de ter uns avós muito presentes, a vários níveis, presentes em tempo, para o trazer, presentes financeiramente que nos apoiam nalgumas coisas, e isto faz a diferença, faz com que nós continuemos a ter alguma qualidade de vida" (M.|filho|4A).

"Realmente os meus pais são dois pilares (...) é o meu pilar e é o pilar do meu C., é fantástico, são mil, o máximo. São fantásticos, fabulosos. Depois tenho também a minha madrinha, o meu padrinho que são como meus segundos pais, que também têm uma ligação ao J. enorme. (...) foram e são, realmente uns pilares muito grandes" ( $\mathrm{H}$.|filho| $8 \mathrm{~A})$.

Apesar da relação entre as mães e os profissionais de saúde nem sempre ser vista como positiva, o apoio das equipas de saúde foi apontado como um contributo importante no processo de adaptação de algumas mães:

"Fomos super bem acolhidos no pediátrico, quando o C. ficou lá internado (...) tivemos a terapeuta no Hospital Pediátrico, que foi assim uma estrelinha fantástica, que fez com que ele, até lá estar, até um ano e meio dois anos, fez o percurso que tinha que fazer como uma criança quase normal" (H.|filho|8A).

Relativamente ao suporte dos profissionais da APC, destaca-se o apoio informativo, pela oportunidade de conhecer melhor a condição, mas também o apoio técnico e prático:

"A mim o que me tem ajudado mais são as terapias, as terapeutas (...) Têm dicas para o ajudar, tem a terapia da fala, como ele não fala, para o ajudar a comunicar, portanto sinto mais as terapeutas que me têm ajudado a saber lidar com a paralisia, a maneira mais fácil, tanto para mim como para ele" (P.|filho|17A).

O tema "Perceção de competência" emerge das descrições das mães sobre a forma como percecionam estar a lidar com êxito com a PC. Esta perceção de competência é evidenciada pela mobilização ativa na reabilitação do(a) filho(a) e na procura de estratégias que potenciem o seu desenvolvimento. $O$ envolvimento das mães é evidente na forma como fazem tudo o que está ao seu alcance para que o(a) filho(a) se desenvolva:

"Mas nós agora vamos tentando tudo, que esta idade chave seja a de maior exploração para que eles consigam abrir um bocadinho mais os campos deles, depois chega a um ponto que já não se desenvolve mais, fica assim mas, até lá, que se consiga o máximo que se conseguir" (C.|filhos|6A).

"Depois acho que me foquei em trabalhar, em poder dar-lhe o melhor em termos da qualidade de vida que ele poderia ter e em termos dos acessos a cuidados de saúde e a mecanismos e a meios, fosse o que fosse. (...). Quero muito ajudá-lo, quero muito que ele tenha... não Ihe vou dizer que não, se eu puder lutar para que ele cada vez arraste menos a perna (M.|filho|4A). 
Por outro lado, a identificação de sinais de evolução dos(as) filhos(as) é também uma força importante para estas mães, contribuindo para a sua confiança na perceção de competência:

"Mas, felizmente, no meu caso tem sido um desafio que tem valido a pena, $e$ uma luta que tem valido a pena. (...).. O meu filho, neste momento escreve. Não tem uma letra tão bonita, não, não tem, mas escreve... e isso para mim é muito gratificante, muito" ( $\mathrm{H}$.|filho| $8 \mathrm{~A})$.

O tema "Pôr a PC 'no seu lugar" reflete a forma como as mães (re)definem o papel que a PC ocupa na vida familiar. Emerge das descrições sobre como procuram evitar centralizar a sua vida em torno da PC e a forma como procuram normalizar a condição. Apesar da PC ser uma condição que comporta muitas exigências no dia-a-dia, as mães também demonstram a necessidade de procurar um equilíbrio, evitando centrar as suas vidas em torno dela:

"Ele tem esta particularidade de precisar de ter estas atenções em algumas coisas, mas isto também não vai ser o foco da nossa família. Já percebemos que se fizermos isso, também descuramos tudo o resto e descuramos daquilo que é o equilíbrio dele que é a família. Ele vai ter a intervenção que nos pudermos pagar, que for prioritária e que não ponha em causa o bem-estar da irmã, também temos isto muito presente. (...). "Estamos a começar a perceber que a família é um todo, não é só o B. não é, e acho que já conseguimos, já atingimos essa maturidade" (M.|filho|4A).

A importância de normalizar o desenvolvimento da criança, apesar das suas limitações, foi também evidente. Assim, uma mãe relata que a severidade ligeira da condição do filho favorece a noção de normalidade:

"O C. é um menino aparentemente normal, ele tem alguma dificuldade na motricidade fina e na fala. É as lacunas do C., ele em termos cognitivos é um menino normal, e até acima da média. Ele está no segundo ano, tem sido uma criança de muito bons, pronto, até agora" (H.|filho|17A).

A comparação com outras crianças com $\mathrm{PC}$ e a valorização do desenvolvimento normal da criança surgem também como uma estratégia de normalização:

"É uma carga de trabalho (risos), porque tenho de explicar à minha filha que tem limitações e dificuldades, mas que há outras crianças iguais a ela, ou piores que ela, e que também não desistem" (C.|filha|15A).

"Eu vou levá-lo, depois espero um bocadinho, vou dando uma volta e vou buscá-lo, para ele poder namorar como os meninos de 16 anos e 17 anos" (I.|filho|17A).

Por fim, o tema "Visão positiva" ilustra a importância de as mães manterem esperança no futuro, bem como a oportunidade de crescimento e mudanças positivas que advém da experiência de cuidar de uma criança/adolescente com PC. Várias mães manifestaram um olhar positivo para o futuro, com esperança na independência e funcionalidade da criança, e na capacidade de ultrapassar as adversidades, apesar das suas limitações:

"Tem de haver sempre esperança e um sentido na vida em dizer assim 'um dia, a minha filha vai tirar a carta, ter carro, ter a própria vida dela e eu voume sentir... feliz"' (R.|filha|15A).

"Eu sei que o C. vai ter sempre complicações, eu sei, mas ele vai, eu acredito que, com a capacidade dele, ele vai conseguir contornar essas situações. Melhor, pior, mas vai conseguir contorná-las, portanto é o que eu espero do futuro" (H.|filho $\mid 8 \mathrm{~A})$. 
Foi também evidenciada a perceção de crescimento e mudanças individuais como resultado da experiência de cuidar de um(a) filho(a) com PC:

"Acho que me sinto mais desenrascada, a tentar resolver os problemas e tudo eu era mais... fechada do que ainda sou hoje, se eu pudesse fugir de tudo, fugia... e eu hoje não fujo das coisas" (I.|filho|17A).

"A mim tornou-me de certeza uma pessoa mais madura, e penso que melhor porque nós na vida achamos que controlamos tudo e o $B$. veio me ensinar que não controlamos nada" (M.|filho|4A).

\section{Discussão}

Este estudo teve como objetivo compreender a experiência de mães de crianças/adolescentes com PC, explorando os fatores que, na sua perceção, contribuem para uma adaptação positiva. A análise das histórias contadas por estas mães permitiu criar um mapa temático que colocou em destaque cinco temas (e respetivos subtemas), que demonstram a importância atribuída à cooperação intrafamiliar, ao papel da rede de apoio, à perceção de competência, à noção de pôr a PC "no seu lugar" e à manutenção de uma visão positiva.

Um dos temas centrais refere-se à importância da cooperação intrafamiliar no processo de adaptação à condição do(a) filho(a). Com efeito, um sentimento de coesão familiar é frequentemente reportado pelas famílias como um fator protetor perante um evento de stress significativo (Patterson, 2002). Neste estudo, destaca-se a colaboração e união do par parental para lidar com a PC, ilustrados pela noção de que o(a) filho(a) é um "projeto comum". Resultados semelhantes foram encontrados por Pelchat et al. (2009) e por Cunha (2011) que evidenciam que, perante uma condição crónica pediátrica, apesar de alguns pais percecionarem o evento como uma fonte de tensão, também emergem mais fortes e unidos. A força que os(as) filhos(as) transmitem às mães também foi identificada como um fator importante para lidar com a PC e, tanto quanto foi possível apurar, emerge como um dado relevante na literatura sobre o processo de adaptação materno à condição.

Relativamente à rede de apoio, as mães também evidenciaram a importância da família alargada. De facto, estudos indicam que o apoio de outros familiares nos cuidados diários à criança (e.g. gestão dos tratamentos) permite diminuir a sobrecarga do cuidador (Reid et al., 2011; Santos et al., 2019), representando fontes de suporte estáveis e de confiança (Gomes et al., 2019). O apoio dos profissionais de saúde foi, também, um recurso muito valorizado, corroborando os resultados do estudo de Reid et al. (2011), que refere que a experiência dos pais é positivamente influenciada pelos profissionais que acompanham a criança. Assim, estes profissionais são considerados uma figura de referência para a família (Santos et al., 2019), devido à prestação de serviços e informações fornecidas, que contribuem para o conhecimento da condição (Reid et al., 2011), bem como pela perceção de acolhimento e vínculo de proximidade (Santos et al., 2019). É interessante notar que, neste estudo, algumas mães que relatam menos apoio familiar, parecem evidenciar maior perceção de apoio por parte dos profissionais de saúde e da APC.

Rolland e Walsh (2006) postulam que, face a uma condição crónica de saúde, a crença de perceção de controlo e competência é também um fator promotor de resiliência. Neste estudo, esta perceção de competência foi particularmente evidenciada pelo destaque de uma atitude proativa dos pais na mobilização para a reabilitação do(a) filho(a), e pela identificação de progressos no seu desenvolvimento. De forma semelhante, Ketelaar et al. (2008) destacam a perceção de competência como importante para os pais de crianças com PC, associado a um maior controlo da adversidade. Por sua vez, Walsh (2016) refere que a resiliência familiar é fortalecida pela experiência e aceitação da passagem do tempo, bem como pelas mudanças que vão surgindo através dos desafios e adversidades. Neste sentido, algumas mães também relatam a perceção de crescimento e mudanças individuais, ilustrando o processo de adaptação à medida que a criança cresce. 
Outro fator importante para a adaptação de algumas mães foi evitar centralizar a sua vida em torno da PC e, assim, "pôr a doença no seu lugar" (González et al., 1989). A tentativa de normalizar a PC, valorizando o desenvolvimento normal da criança e desenvolvendo esforços para que esta não se sinta diferente, emerge no âmbito deste tema, indo ao encontro dos estudos de Cunha (2011) e Rehm \& Bradley (2005). Estes autores apontam também o foco no dia-a-dia, a comparação com outras situações e a minimização do impacto da doença na vida familiar como estratégias para relativizar a condição de saúde do(a) filho(a).

Por fim, a manutenção de uma visão positiva e de esperança no futuro, sobretudo no que refere à independência e funcionalidade da criança, foi também identificado como um tema relevante. Estes resultados são consistentes com os processos chave de resiliência familiares propostos por Walsh (2016), ao enfatizar que a esperança e o otimismo promovem a capacidade da família para enfrentar e recuperar de situações adversas.

\section{Conclusão}

Este estudo apresenta algumas limitações, como o facto de incluir apenas mães. Assim, seria importante que, em estudos futuros, pais do sexo masculino estejam representados, por forma a aprofundar, também, as experiências paternas. Acresce que o reduzido número de participantes no estudo não permitiu que a saturação teórica dos dados fosse atingida. Futuramente, poderá ser interessante estudar amostras mais diversificadas, na qual se encontrem representadas diferentes características, quer dos pais, quer das crianças, bem como estender a unidade de análise a outros elementos do sistema familiar, para melhor compreender a sua experiência no processo de adaptação à PC. Seria ainda importante estudar a articulação entre os desafios (impacto) e a experiência de adaptação destas famílias,

Não obstante estas limitações, os resultados permitem evidenciar que, apesar dos desafios inerentes à PC, as mães conseguem mobilizar recursos e ativar forças ao longo da sua experiência. Assim, a avaliação e a intervenção com estas famílias devem procurar expandir as suas competências e recursos, de modo a promover a resiliência familiar e a fortalecer os laços relacionais entre os membros da família.

\section{Referências}

Braun, V., \& Clarke, V. (2006). Using thematic analysis in psychology. Qualitative research in psychology, 3, 77-101. https://doi.org/10.1057/978-1-137-35913-1.

Cunha, A. I. (2011). Histórias e trajetórias de adaptação e resiliência familiar na doença crónica pediátrica (Tese de Doutoramento). Retirado do repositório científico da Universidade de Coimbra (http://hdl.handle.net/10316/20310).

Cunha, K. da C., Ramos, M. F. H., Silva, S. S. da C., \& Pontes, F. A. R. (2017). Estresse parental e paralisia cerebral. Psicologia, Saúde e Doenças, 18(1), $433-450$. http://doi.org/10.15309/17psd180212.

Dixon-Wood, M., Young, B., \& Heney, D. (2002). Childhood cancer and users' views: A critical perspective. European Journal of Cancer Care, 11, 173-177. https://doi.org/10.1046/j.13652354.2002.00335.x.

Eunson, P. (2016). Aetiology and epidemiology of cerebral palsy. Paediatrics and Child Health (United Kingdom), 26(9), 367-372. https://doi.org/10.1016/j.paed.2016.04.011.

Gomes, G. C., Jung, B. C. de, Nobre, C. M. G., Norberg, P. K. de O., Hirsch, C. D., \& Dresch, F. D (2019). Rede de apoio social da família para o cuidado da criança com paralisia cerebral. Revista Enfermagem UERJ, 27, e40274. http://doi.org/10.12957/reuerj.2019.40274.

González, S., Steinglass, P., \& Reiss, D. (1989). Putting the illness in its place: Discussion groups for families with chronic medical illnesses. Family Process, 28(1), 69-87. https://doi.org/10.1111/j.1545-5300.1989.00069.x. 
Guillamón, N., Nieto, R., Pousada, M., Redolar, D., Muñoz, E., Hernández, E., ... Gómez-Zúñiga, B. (2013). Quality of life and mental health among parents of children with cerebral palsy: The influence of self-efficacy and coping strategies. Journal of Clinical Nursing, 22(11-12), 15791590. https://doi.org/10.1111/jocn.12124.

Ketelaar, M., Volman, M. J. M., Gorter, J. W., \& Vermeer, A. (2008). Stress in parents of children with cerebral palsy: What sources of stress are we talking about? Child: Care, Health and Development, 34(6), 825-829. https://doi.org/10.1111/j.1365-2214.2008.00876.x.

Macedo, E. C., Da Silva, L. R., Paiva, M. S., \& Ramos, M. N. P. (2015). Sobrecarga e qualidade de vida de mães de crianças e adolescentes com doença crônica: Revisão integrativa. Revista Latino-Americana de Enfermagem, 23(4), 769-777. https://doi.org/10.1590/01041169.0196.2613.

Palisano, R., Rosenbaum, P., Bartlett, D., \& Livingston, M. (2008). Content validity of the expanded and revised Gross Motor Function Classification System. Developmental Medicine \& Child Neurology, 50(10), 744-750. http://dx.doi.org/10.1111/j.1469-8749.2008.03089.x.

Patterson, J. M. (2002). Understanding family resilience. Journal of Clinical Psychology, 58(3), 233246. https://doi.org/10.1002/jclp.10019.

Pelchat, D., Levert, M. J., \& Bourgeois-Guérin, V. (2009). How do mothers and fathers who have a child with a disability describe their adaptation/transformation process? Journal of Child Health Care, 13(3), 239-259. https://doi.org/10.1177/1367493509336684.

Pousada, M., Guillamón, N., Hernández-Encuentra, E., Muñoz, E., Redolar, D., Boixadós, M., \& Gómez-Zúñiga, B. (2013). Impact of caring for a child with cerebral palsy on the quality of life of parents: A Systematic Review of the Literature. In Journal of Developmental and Physical Disabilities (Vol. 25). https://doi.org/10.1007/s10882-013- 9332-6.

Raina, P., Rosenbaum, P., Brehaut, J., Walter, S. D., Russell, D., Swinton, M., ... Wood, E. (2005) The health and well-being of caregivers of children with cerebral palsy. Pediatrics, 115(6), 626635. https://doi.org/10.1542/peds.2004-1689.

Rehm, R. S., \& Bradley, J. F. (2005). Normalization in families raising a child who is medically fragile/technology dependent and developmentally delayed. Qualitative Health Research, 15(6), 807-820. https://doi.org/10.1177/1049732305276754.

Reid, A., Imrie, H., Brouwer, E., Clutton, S., Evans, J., Russell, D., \& Bartlett, D. (2011). "If i knew then what i know now": Parents' reflections on raising a child with cerebral palsy. Physical and Occupational Therapy in Pediatrics, 31(2), 169-183. https://doi.org/10.3109/01942638.2010.540311.

Rolland, J. S., \& Walsh, F. (2006). Facilitating family resilience with childhood illness and disability. Current Opinion in Pediatrics, 527-538. https://doi.org/10.1097/01.mop.0000245354.83454.68.

Santos, A. A. de S., Oliveira, C. C. da C., Vargas, M. M., \& Macedo, I. D. A. B. (2010). Avaliação da sobrecarga dos cuidadores de crianças com paralisia cerebral. Ciência, Cuidado e Saúde, 9(3). 503-509. https://doi.org/10.4025/cienccuidsaude.v9i3.9405.

Santos, B. A., Milbrath, V. M., Freitag, V. L., Gabatz, R. I. B., \& Vaz, J. C. (2019). Rede de apoio social à família da criança com paralisia cerebral. Revista de Pesquisa: Cuidado é Fundamental Online, 11(5), 1300-1306. https://doi.org/10.9789/2175-5361.2019.v11i5.13001306.

Santos, B. A., Milbrath, V. M., Freitag, V. L., Nunes, N. J. da S., Gabatz, R. I. B., \& Silva, M. S. da. (2019). The impact of cerebral palsy diagnosis from the perspective of the camily. Reme Revista Mineira de Enfermagem, 23. https://doi.org/10.5935/1415-2762.20190035.

Walsh, F. (2016). Family resilience: A developmental systems framework. European Journal of Developmental Psychology, 13(3), 313-324. https://doi.org/10.1080/17405629.2016.1154035. 